\title{
Regulation of targeting determinants in interorganelle communication
}

\author{
Ganiyu B Alli-Balogun and Tim P Levine* \\ UCL Institute of Ophthalmology, 11-43 Bath Street, London EC1V 9EL \\ * Correspondence to tim.levine@ucl.ac.uk
}

\begin{abstract}
The field of interorganelle communication is now established as a major aspect of intracellular organisation, with a profusion of material and signals exchanged between organelles. One way to address interorganelle communication is to study the interactions of the proteins involved, particularly targeting interactions, which are a key way to regulate activity. While most peripheral membrane proteins have single determinants for membrane targeting, proteins involved in interorganelle communication have more than one such determinant, sometimes as many as four, as in Vps13. Here we review the targeting determinants, showing how they can be relatively hard to find, how they are regulated, and how proteins integrate information from multiple targeting determinants.
\end{abstract}

\section{Keywords}

membrane contact sites; interorganelle communication; lipid traffic calcium signaling; amphipathic helix; VPS13; VAP; ATG2

\section{Running Title}

Targeting for interorganelle communication

Word Count: 2502 


\section{Highlights}

- proteins target two organelles at the same time to form bridges between them

- targeting is often by short regions such as motifs and amphipathic helices

- the only widespread way to target the ER is via a FFAT motif that binds VAP

- FFAT motifs can be both created and destroyed by phosphorylation

- amphipathic helices, often highly specific for one organelle, are hard to find 


\section{Introduction}

Organelles compartmentalise biochemical reactions, but they do not function in isolation. Interorganelle communication takes place by direct pair-wise contact, which is mediated by protein bridges that communicate many activities, including signal transduction [1], trafficking second messengers such as calcium ions $\left(\mathrm{Ca}^{2+}\right)$ [2], cyclic AMP [3], reactive oxygen species [4], and other small molecules including

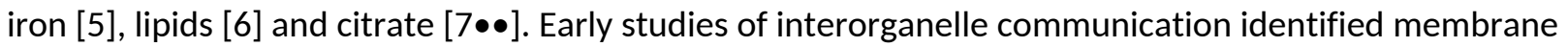
contact sites where different membranes come close together indicating communication. Most of these organelles are separated by gaps of 10-30 nm, compatible with being bridged by a single protein, though there are notable exceptions [8,9]. Now the field has matured, so the question is not which organelle pairs interact, but identifying a pair that does not $[7 \bullet \bullet]$.

Proteins that bridge between organelles must include two targeting determinants to bind two receptors, so they can be in two places at the same time [10]. This pattern is exemplified by oxysterol binding protein (OSBP)-related proteins (ORPs) such as ORP5, with an N-terminal pleckstrin homology (PH) domain binding $\mathrm{PI}(4,5) \mathrm{P}_{2}$ on the plasma membrane, and a C-terminal transmembrane helix (TMH) anchored in the endoplasmic reticulum (ER) [11]. A bridging protein's action derives from the nontargeting domain(s), which here is a lipid transfer domain (Figure 1A).

This review addresses targeting determinants involved in interorganelle communication (Figure 1B-D), their regulation by post-translational modification and intramolecular inhibition, and how they integrate together, especially in proteins with more than two determinants, such as VPS13.

\section{FFAT motifs link the ER to most organelles}

The protein element most widely involved in communication between organelles is the "two phenylalanines in an acidic tract" (FFAT) motif. Its core is seven residues in a loop that binds to the globular domain of Vesicle-associated membrane protein-Associated Proteins (VAPs) on the cytoplasmic face of the ER (Figure 1D). There are three VAPs: VAP-A and VAP-B, which are highly similar, and the less related MOSPD2 (details below). High-throughput studies in human cells have identified a large number (>300) of VAP interactors, many with functions in, and targeting domains/motifs for, organelles other than the ER $[12,13]$. If the high-throughput VAP interactome could be verified, many new pathways would be implicated in interorganelle communication. For example, several proteins that scaffold and regulate protein kinase $A$ are included in the VAP interactome; verifying this would fits with other findings that show involvement of the ER in CAMP signaling [3].

To bind VAP, FFAT motifs combine several residues in the core to create specific "key in lock" recognition, while the acidic tract upstream provides charge-based, non-specific affinity $[14,15]$. Identical FFAT motifs were originally found in three families of lipid transfer proteins (Figure 2A) [16], but FFAT motifs have since turned out to be quite variable, in particular at position 3 (Figure 2B) $[17,18]$. Among the various pathways that access the ER via VAP, lipid traffic has a special relationship, as indicated by the domain composition MOSPD2. The C-terminus of this third VAP homologue present 
throughout the animal kingdom binds FFAT motifs just like VAP-A/B. However, its N-terminus is extended to include a lipid transfer domain, suggesting an intimate association between VAP and lipid traffic [19•].

\section{Similar motifs indicate dozens more interorganelle pathways}

Within FFAT motifs positions $2 / 4 / 5$ vary the least, and a bioinformatic search in all human VAP interactors identified motifs significantly similar to FFAT in 24 VAP interactors [20], but experimental validation is needed. So far, 3 out of these potential FFAT-VAP interactions have been validated: SNX2 on endosomes [21], ACBD5/4 on peroxisomes [22-24], and VPS13A/C. VPS13 is a family of large (>3000 aa) conserved proteins with one homologue in yeast, four in mammals $(A / B / C / D)$, and three in flies $(A / B / D)[25 \bullet \bullet]$. A ground-breaking study has identified the $\mathrm{N}$-terminus as a lipid transfer protein [26••]. Together with single particle analysis showing that VPS13 is a long rod [27•], this suggests that it forms static bridges across which lipid flows, as are found in bacteria (Figure 2C) [28]. VPS13A and VPS13C both have FFAT-like motifs [26••], and multiple other membrane targeting determinants (see below).

The importance of the FFAT-VAP interaction is indicated by it being parasitised by intracellular pathogens $[29,30]$. In one case, a bacterially-encoded protein named IncV for inclusion protein

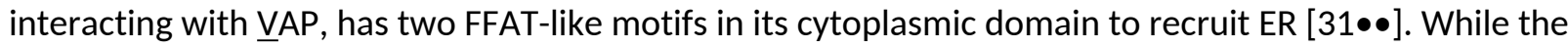
motifs described in all these proteins do not meet the original definition of FFAT, they do bind VAP, which indicates that the definition of the motif should be enlarged to include any sequence with sufficient features, especially at positions 2,4 and 5, even with only 1 phenylalanine (or tyrosine) or without an acidic tract.

\section{Modifying FFAT motifs to regulate interorganelle communication}

Intracellular communication can be regulated by phosphorylation in VAP interactors that have serines or threonines at the positions otherwise assumed by acids: core \#1/4/7 and the whole upstream tract (Figure 2B) [16]. For example, in AKAP3 phosphomimetic substitution of T4 with D4 enhances binding in vivo, while A4 inhibits [20], and the same likely applies to StARD3 on endosomes [32] and norovirus nonstructural-1/2 (NS1/2) protein [33•]. Ceramide transferase (CERT), a FFAT+ve lipid transfer protein with similar domain structure to OSBP (Figure $2 \mathrm{~A}$ ), is phosphorylated in its acidic tract, which strongly enhances VAP binding (Figure 2B, and section below) [34]. FFAT motifs can be phosphorylated multiple times, as shown by Kv2.1 and Kv2.2 (collectively Kv2), potassium voltage-gated channels that regulate neuronal excitability. Kv2 forms inactive plasma membrane clusters during repetitive stimulation $[35,36]$. Clustering takes just a few minutes, requiring multiple serine phosphorylation in Kv2's cytoplasmic tail $[37,38]$, and is reversed by dephosphorylation [35,39]. After Kv2 clusters were found recruit cisternae of cortical ER [38], the next step was to identify an essential phenylalanine among the phosphoserines, and this led to finding that Kv2 contains an inducible FFAT motif that underlies the

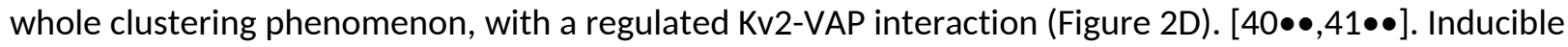


FFAT motifs are conserved in Kv2 across evolution indicating a fundamental link between excitability and the ER [36].

In contrast to activating phosphorylation at position 4, phosphorylation at position 5 completely blocks VAP binding [20]. VAP interactors with S5/T5 that might be inactivated by phosphorylation include JMY, which nucleates actin at the TGN [42] and ACBD5/ACBD4 in peroxisomal lipid traffic [23,24]. Reversible sulphenylation by reactive oxidising species such as peroxide interacting with cysteine at position 5 in would be expected to negatively regulate many FFAT motifs [43,44]. Although not yet untested, this would allow intracellular redox signaling to inhibit VAP binding, for example during the reactive oxygen species burst during calcium signaling [4]. The significance of C5 can be gauged by its widespread conservation in both Kv2 (Figure 2D) and Rab3GAP1 (not shown), indicating functional selection.

In addition to regulation via FFAT motifs, VAP is modified. In yeast, VAP's linker is reversibly sumoylated, though the functional consequences are unknown (Figure 1D) [45]. In humans, lysine acetylation on the FFAT-binding surface of VAP-A (but not VAP-B) occurs in response to viral infection [46]. Such posttranslational modifications would alter intracellular communication globally [44].

\section{Charged based regulation by phosphorylation}

Targeting of peripheral membrane proteins can be based on simple physical properties such as charge. For example inherently disordered polybasic regions have non-specific affinity for the polyanionic plasma membrane (Figure 1B) [47]. The integral ER lipid transfer protein TMEM24 has a long cytoplasmic tail ( 400aa) that contains three disordered polybasic stretches ( $\sim 80$ aa). The key here is that this region is also enriched for serines and threonines. Phosphorylation by protein kinase $\mathrm{C}$ and dephosphorylation by protein phosphatase $2 \mathrm{~B}$ respectively inactivate/activate TMEM24 in pancreatic $\beta$ cells by reducing/increasing the overall positive charge of the bridging region, leading to rapid cycling that facilitates episodic insulin secretion [48••].

Other polybasic regions can reversibly fold into amphipathic helices, where one face is hydrophilic (positively charged), and the opposite face is hydrophobic, penetrating the hydrophobic portion of a bilayer (Figure 1C). Amphipathic helices have specificity for a unique organelle, including the ER, by detecting combinations of charge and packing (curvature and acyl chain composition) [49-51]. Ice2p, an integral yeast ER membrane protein of unknown function, bridges to lipid droplets via lipid dropletspecific amphipathic helices (highly hydrophobic, without net charge) [52]. In contrast, another yeast integral ER protein, Ist2p, bridges to the plasma membrane via an amphipathic helix with properties suited to that membrane (minimally hydrophobic, highly basic) [53]. Many of nine serines/threonines adjacent to Ist2p's helix can be phosphorylated [54], indicating a possible (though untested) means of regulating bridging. 


\section{Co-ordinated regulation by other domains in cis}

Membrane targeting can be regulated by intramolecular interactions, which dominate because of the extremely high local concentration of domains linked in cis. For integral ER membrane proteins, such as STIM1[55,56] and extended-synaptotagmin-1 (E-Syt1) [57••], activation is associated with translocation from the whole organelle to interorganelle bridges only. In contrast, lipid transfer proteins such as CERT and ORPs have inactive forms that are diffuse in the cytoplasm, indicating that both targeting determinants are masked (Figure 2B). Co-ordinated switching of both determinants has been demonstrated for CERT. CERT is maximally active when the acidic tract of the FFAT is phosphorylated [33], and a serine rich motif (SRM) adjacent to the PH domain is fully dephosphorylated (Figure 3B left, and Figure 2B) [58]. In this state, all the domains are active. Multiple phosphorylation of the SRM leads

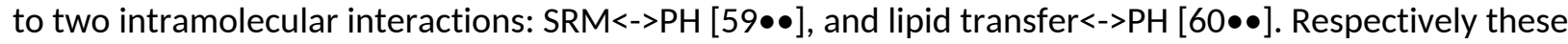
inhibit TGN targeting and occlude lipid transfer. Affinity for VAP drops further with dephosphorylation of the FFAT (Figure 3B, right). The SRM is part of a homeostatic loop: lipid transfer by CERT leads to production of diacylglycerol in the TGN, which activates protein kinase D. This initiates phosphorylation of the SRM, which initiates repeated action of Casein Kinase I. The multiply phosphorylated SRM inhibits CERT, thus reducing diacylglycerol production. Subsequently the SRM is reactivated by the integral ER protein phosphatase $2 \mathrm{C} \varepsilon$ [61]. Since protein phosphatase $2 \mathrm{C} \varepsilon$ binds to VAP (their TMH regions interact), CERT appears to bind VAP before it is activated, suggesting that its FFAT motif is partly active even in the inactive/closed form (Figure 3, right).

The domain structure of ORPs is similar to CERT (Figure 2A), and like CERT, lipid transfer by OSBP is regulated by other domains in cis [62]. OSBP's lipid transfer domain is inhibited by the other domains, and VAP binding to OSBP's FFAT relieves the inhibition, irrespective of membrane targeting by VAP [62]. This implies that VAP could be considered as an accessory subunit of an extended lipid transfer device, not merely an anchor. One proposed mode of action for VAP is through oligomerisation, as it (like CERT and OSBP) has a short coiled-coil and other elements that mediate oligomerisation [15]. Another possibility is that VAP dimers bring together two unrelated FFAT-positive proteins. This may explain how SNX2 recruits OSBP to endocytic tubules rather than its usual TGN localisation [21].

\section{Integrating three or more targeting motifs in one protein}

In addition to bridging proteins with two targeting determinants, there are proteins with three or more targeting determinants. Consideration of these requires expansion of the simple model of bridging proteins targeting each organelle once only.

\section{One interaction repeated twice:}

There are several proteins with two functional FFAT motifs. IncV has two adjacent motifs, with the double mutation required to ablate VAP recruitment [31]. SNX2 has two FFAT motifs, but here one motif has a dominant effect on VAP interaction [21]. Maybe the second motif allows increased avidity for VAP. However, this may be an over-simplification, since ORP3 has a motif almost identical to that of OSBP (Figure 2A), but surprisingly a highly variant motif adjacent to the $\mathrm{PH}$ domain has to be mutated too completely disrupt VAP binding [63]. 


\section{Same organelle twice:}

Some proteins combine two different determinants to target the ER. For example, Protrudin has TMHs for permanent ER targeting, a PX domain to bridge to endosomes, and a FFAT motif which functions to regulate its distribution throughout the ER $[18,64]$. This suggests that bridging complexes alter their distribution (and function) by incorporation of additional subunits.

\section{Targeting motifs for 3 organelles by VPS13:}

We described above how VPS13A bridges from ER to lipid droplets (Figure 2D, right). It also contains other targeting determinants that have recently been mapped (Figure $4 \mathrm{~A}$ ). The overall domain structure of VPS13A/C in animals is similar to Vps13p in yeast, but their targeting is divergent.

In yeast, Vps13p acts at multiple locations: (i) mitochondria, (ii) prospore membranes - specific to meiosis, (iii) endosome/vacuole (equivalent of lysosome) - varying from endosomal puncta in log phase to nucleus-vacuole junction in stationary phase (Figure 4B, left) [65]. All of these share a single mode of targeting. A highly conserved region in the C-terminal half of Vps13p (so-called DUF1162, also predicted to be a WD40-like beta propeller) binds an unstructured "PxP" motif shared by three receptors, one per target organelle: Mcp1p on mitochondria, Spo71p on prospore membranes, and Ypt35p (a PX domainonly protein homologous to sorting nexins) on endosome/lysosomes. The three receptors competing for a common binding site on $\mathrm{Vps13p}$ [66••]. The targeting of $\mathrm{Vps13p}$ to the nucleus-vacuole junction requires vacuolar Ypt35p and an as yet unidentified ER receptor (Figure 4B, left) [66]. The same receptor may allow Vps13p to bridge from the ER to mitochondria and prospore membranes.

In mammals two duplications have created parologous pairs VPS13A/C and VPS13B/D. VPS13A/C have FFAT motifs and PH domains (Figure 4A). Not much is known about VPS13B/D targeting, although VPS13D has a short ubiquitin-associated (UBA) domain involved in mitophagy [25]. VPS13A and VPS13C both have targeting determinants for a third organelle: VPS13A for mitochondria; VPS13C for late endosomes/lysosomes (Figure 4B) [26••]. It is not known if VPS13C's endosomal receptor binds similarly to Ypt35p binding Vps13p. The FFAT motifs work simultaneously with either of the other determinants to target VPS13A/C to interorganelle junctions (Figure 4B, right).

How do the multiple targeting determinants work together? Given the position of all the non-ER targeting determinants in the C-terminus of VPS13A/C and Vps13p, it may not bridge between non-ER organelles. Therefore, suggestions that Vps13p bridges directly from vacuole to mitochondria at vacuole and mitochondrial patches (VCLAMPs) [67••] may have to be revised in favour of an indirect mechanism that combines both vacuole-ER and mitochondria-ER bridges. This is supported by finding Vps13p adjacent to, but not coincident with, vCLAMPs [68••].

\section{Conclusions and future directions}

The quality of intracellular targeting by proteins involved in interorganelle communication is like other proteins, but the quantity is greater: often twice as much, and in some cases more, in particular VPS13. Future questions include how to identify all motifs and regions responsible for targeting, with shorter sequences being harder to find than whole domains. This may explain the missing targeting 
determinants in key proteins such as Vps13p: how does it target the ER? It may be that Vps13p, VPS13B/D and other players in intracellular communication have amphipathic helices, so we will need proteome-wide maps of amphipathic helices.

\title{
Acknowledgements
}

We acknowledge support from the BBSRC (BB/P003818/1).

\section{Disclosure}

The authors declare no conflict of interest.

\begin{abstract}
Abbreviations
extended-synaptotagmin - E-Syt; two phenylalanines in an acidic tract - FFAT; non-structural-1/2 protein (in norovirus) - NS1/2, oxysterol binding protein - OSBP; OSBP Related Protein - ORP5; pleckstrin homology - PH; synaptotagmin mitochondrial and lipid binding domain - SMP domain; steroidogenic acute regulatory lipid transfer protein - StART; transmembrane helix - TMH; trans Golgi network - TGN; vesicle-associated membrane protein-associated protein - VAP
\end{abstract}

\section{Legends}

Figure 1: Short motifs involved in interorganelle bridging.

A. Proteins involved in interorganelle communication typically have targeting determinants for two different organelles. Oxysterol binding protein (OSBP)-Related Protein-5 (ORP5) has an N-terminal pleckstrin homology $(\mathrm{PH})$ domain that binds $\mathrm{PI}(4,5) \mathrm{P}_{2}$ to target the plasma membrane (yellow), and a Cterminal transmembrane helix (TMH) anchored in the ER (ochre). Between these two domains is a lipid transfer domain that can shuttle (yellow arrow) from the PI4P from the plasma membrane, where it is made, to the ER, where it is destroyed. ORP5 moves a second lipid, phosphatidylserine, from ER to plasma membrane, which is omitted for clarity. B-D. Different ways short peptide stretches reversibly target proteins involved in interorganelle communication. B. Non-specific charge attraction between unstructured regions rich in basic residues (blue circles) and anionic phospholipids, mainly phosphatidylserine (red circles). This is found in TMEM24. Switching is achieved by multiple phosphorylations particularly of multiple serines and threonines (circles with orange border $\Leftrightarrow$ filled with red) within the polybasic regions. C. Unstructured regions containing many hydrophilic residues (here basic - blue) and hydrophobic residues (yellow, $\leq 30 \%$ ), as in Ist2p, are induced to fold into an amphipathic helix and partially insert into plasma membrane. Alternate choice of hydrophilic residues (more uncharged, purple) and increased hydrophobic residues changes targeting (see Figure $2 \mathrm{C}$ ). 
Amphipathic helices can be switched by phosphorylation of residues within the helix or adjacent to it. D. Short linear motifs recognise integral membrane proteins. Here a FFAT motif (red) is shown engaging with VAP. Switching is achieved by phosphorylation or intramolecular masking of the FFAT motif. VAP is also subject to post-translational modification, for example sumoylation of a conserved site (asterisk), the effect of which is unknown. For clarity, the coiled coil in the linker for VAP has been omitted.

Figure 2: Multiple pathways access the ER by FFAT motifs and variants that bind VAP.

A. Canonical FFAT motifs occur mainly in three families of lipid transfer proteins: oxysterol binding protein (OSBP), retinal degeneration type $B$ ( $R d g B$, homologous to the human proteins Nir2/3), and ceramide transferase (CERT). Globular domains are as in Figure 1A, plus those indicated in RdgB. One dimensional domain maps, with FFAT motifs (red). B. FFAT motifs, one canonical (EFFDAxE) in CERT and two non-canonical in FAF1, and protrudin. 6 residues in the core of 7 are subject to selection (black background), as is the $\mathrm{N}$-terminal acidic tract ( 6 residues). Colouring of residues: $\mathrm{F} / \mathrm{Y}$ at positions $2 / 3$ of the core - yellow; acids - red; bases - blue; small side-chain for positon 5 (ACGSP) - green; S/T in positions that prefer acids - orange; unselected core position 6 - black; all others - grey. Red box and asterisk (CERT S315) indicates where phosphorylation has been demonstrated in vivo. C. A predicted non-canonical FFAT motif has been verified experimentally in VPS13A, which forms a rod-like lipid transfer protein $\sim 20 \mathrm{~nm}$ long with a semi-circular side-arm [26,27]. Its C-terminus includes an amphipathic helix that targets lipid droplets, as it is enriched in hydrophobic residues and not highly basic, unlike helices that select for plasma membrane (Figure 1C). FFAT motif below coloured as in B. VPS13C is highly similar, with a longer predicted rod domain. D. Non-canonical FFAT motifs are induced by multiple phosphorylation in Kv2.1 (and Kv2.2, not shown), which bridges from the plasma membrane to VAP in the ER. Red boxes indicate residues that are phosphorylated, and asterisks indicate residues essential for reversible clustering. The sequence from Kv2 in a mollusc, pacific oyster (Crassostrea gigas), shows conservation of this pattern across animal evolution. Drawing is approximately to scale in (C), with a $20 \mathrm{~nm}$ gap between membranes and unstructured loops at $0.38 \mathrm{~nm}$ per peptide bond. Parts of the cytoplasmic tail of Kv2.1 before and after the FFAT motif (170 and 260 residues respectively) are omitted in (D). For clarity, coiled coils, for example in the linker for VAP, are omitted.

Figure 3: Intramolecular interactions that regulate ceramide traffic by CERT

Inducible intramolecular inhibitory interactions regulate CERT, which contains a pleckstrin homology (PH) domain, serine rich motif (SRM), FFAT motif, and steroidogenic acute regulatory lipid transfer (StART) domain, asterisks indicating phosphorylation sites. TOP: one dimensional domain maps indicating intramolecular inhibitory interactions; BOTTOM: two dimensional representations of the domains and loops in interorganelle gaps, showing inter-domain binding. When active, CERT adopts an open conformation, with the PH domain targeting the TGN by coincidence detection of Arf1 (not shown) and PI4P, and the FFAT motif (especially when phosphorylated on S315, FFAT*, see Figure 2B) targeting VAP on the ER. The StART domain can shuttle lipid across the gap (ceramide forward from the ER, 
possibly also carrying diacylglycerol in the opposite direction). The SRM with zero phosphorylations $\left(S M^{0}\right)$ has no discernible effect. Fully phosphorylated SRM $\left(S^{2} M^{10}\right)$ induces an inhibitory interaction between the PH and StART domains, and it directly binds and inhibits the PH domain. Under these circumstances, the FFAT motif is dephosphorylated (FFAT ${ }^{\circ}$ ), and affinity for VAP decreases, though not to zero. The ER-TGN gap is drawn at $20 \mathrm{~nm}$, but may be narrower [69].

Figure 4: VPS13 integrates multiple targeting determinants with specificity for four different organelles

A. Domain map of VPS13A, showing all unstructured loops $\geq 40$ aa, including those within the rod domain, and three C-terminal domains: DUF1162/WD40 of $\leq 8$ WD40-like repeats; Dbl Homology domain-like $\left(\mathrm{DH}_{\mathrm{L}}\right)$ that includes an amphipathic helix; $\mathrm{PH}$. VPS13C has an $\mathrm{N}$-terminal internal duplication, so likely forms a longer rod-like lipid transfer domain. Vps13p is similar in form, but has no FFAT motif.

ATG2 is likely to be similar, as it is homologous to VPS13 at both ends and also forms rods [70]. B. VPS13 forms a $20 \mathrm{~nm}$ rod-like lipid transfer protein with an incomplete loop side-arm, which is here presumed to contain the C-terminal targeting determinants, coloured as in (A). Black arrows indicate organelle targeting, and yellow arrows indicate interorganelle communication. LEFT. Yeast Vps13p.

DUF1162/WD40 binds receptors on mitochondria, prospore membrane and endosome/vacuole (Mcp1p, Spo71p, Ypt35p respectively, each containing related "PxP" motifs). Vps13p targets the nucleus-vacuole junction (NVJ), indicating that it has an ER receptor, which has yet to be mapped. In prospore membrane biogenesis, it is not clear if $\mathrm{Vps} 13 \mathrm{p}$ bridges to a second membrane, as the protein surrounds the entire prospore uniformly [65]. Vps13 interacting with mitochondria is adjacent to vacuole and mitochondrial patches (vCLAMPs), so rather than directly bridging between these organelles, it might form bridges from both to the ER [68••]. RIGHT: Human VPS13A/C. FFAT motifs are found in middle of the rod-like portion. The C-termini target to two other organelles: DUF1162/WD40 of VPS13C targets late endosomes; both $\mathrm{DH}_{\mathrm{L}}$ and PH of VPS13A target mitochondria. An amphipathic helix in both proteins targets lipid droplets. Targeting by the C-terminus occurs simultaneously with ER targeting: in VPS13C at late endosomal-ER junctions (LE-ERJ), in VPS13A at mitochondrial-ER junctions (M-ERJ), and in both at lipid droplet-ER junctions (LD-ERJ).

\section{References and recommended reading}

Papers of particular interest, published within the period of review, have been highlighted as:

- of special interest $\bigotimes$

$\bullet$ of outstanding interest

\section{Text on references of interest:}

7. $\bullet$ Shai 
In this study, the authors used split-fluorescence reporter systems to study six organelles for direct communication between each other (peroxisomes, mitochondria, plasma membrane, vacuole, ER and lipid droplets), and all 15 possible pairs showed bridging interactions. Mitochondria-peroxisome bridges were studied in detail, with two proteins shown to tether the organelles: a pool of peroxisomal Fzo1p, a yeast mitofusin homologue, which possibly bridges by homotypic interaction with mitochondrial Fzo1p, and peroxisomal Pex34p, which is shown to be linked to transport of citrate across the contacts with mitochondria.

19 Di Mattia

This study shows that MOSPD2 is the third VAP, with a major sperm (MSP) domain equally able to bind VAP as in VAP-A and VAP-B, and an additional lipid transfer domain. In cells that express MOSPD2, triple knockdown has a stronger phenotype on the extent of some interorganelle contacts than VAPA/Bdouble knockdown.

$25 \bullet \bullet$ Anding

To better understand mitophagy, a screen of $>100$ ubiquitin-binding proteins in flies for their role in a growth stage characterised by mitochondrial clearance produces three hits, one being VPS13D included in the screen because of a polyubiquitin binding UBA domain in its C-terminus. The role of VPS13D in driving mitophagy to control mitochondrial morphology is conserved in human cells

\section{$26 \bullet \bullet$ Kumar}

This paper reveals that VPS13A/C are lipid transfer proteins with a large lipid transfer domain (both long and wide), which is predicted to form a static rod-like structure along which multiple lipid molecules flow. Targeting domains are mapped for the ER, lipid droplets and mitochondria/late endosomes. This work challenges the paradigm for intracellular lipid traffic by demonstrating a large number of lipid transfer proteins (superfamily includes ATG2) that are predicted to lack of specificity for lipid headgroup.

27/70 • De / Chowdhury

These two papers use single particle electron microscopy to show that both Vps13p (yeast) and ATG2A (human) are long rods, with ATG2A binding membrane at each end, allowing it to bridge between organelles. Given that the N-terminus of VPS13 is a lipid transfer protein (see note ref [26]), and the homology between the N-termini of VPS13 and ATG2, and this suggests VPS13 and ATG2 are a new superfamily of eukaryotic bridge-like lipid transfer proteins similar to LptCAD in bacteria.

$31 \cdot$ Stanhope

Here a previously undescribed protein on the inclusions of the intracellular bacterial pathogen Chlamydia trachomatis IncV is shown to recruit the ER by binding directly to VAP, leading to it being called IncV.

$33 \cdot$ McCune 
The interaction of non-structural protein NS1/2 of murine norovirus with VAP is shown to be required for viral replication. The precise binding site on NS1/2 is mapped to a sequence that bears only a remote resemblance to the FFAT motifs originally described in OSBP, CERT and RdgB (Nir2/3 in humans).

40/41 •• Johnson/Kirmiz

The molecular basis of clustering of the voltage-gated $\mathrm{K}^{+}$-channels Kv2.1 and Kv2.2 in the plasma membrane is explained (more than 25 years its discovery) by a covert FFAT motif. Clustered Kv2, previously known to that recruit cortical ER cisternae, is shown to bind directly to VAP via FFAT-like motifs induced by multiple $\mathrm{S} / \mathrm{T}$ phosphorylations and a conserved phenylalanine previously shown to be essential for clustering. The absolute requirement for $\mathrm{Kv} 2$ in bringing the two organelles together, and the lack of any other mechanism by which other domains in Kv2 might affect organelle architecture, makes Kv2 one of the best examples of a tether in interorganelle communication.

$48 \bullet \bullet$ Lees

Insulin secretion is linked to lipid transfer replenishing plasma membrane phosphatidylinositol by the lipid transfer protein TMEM24 which is anchored in the ER. Insulin secretion events cause raised cytoplasmic calcium which leads to inactivating phosphorylation of TMEM24. Following secretion, TMEM24 dephosphorylated by calcineurin replenishes the precursor of this lipid to allow another cycle of secretion. The solved structure of the lipid transfer domain indicates it acts as a shuttle not as a tube.

\section{$57 \bullet \bullet B i a n$}

The authors elucidate the molecular mechanisms of calcium mediated localisation of E-Syt1 within the ER to cortical sites adjacent to the plasma membrane showing that both of the two C2 domains in E-Syt1 that can bind calcium have (in its absence) inhibitory interactions with other domains in E-Syt1, these being relieved when cytoplasmic calcium rises, temporarily activating E-Syt1. The calcium effect applies to functions of E-Syt1, both transfer of the lipid diacylglycerol from the plasma membrane, and the enabling scrambling of plasma membrane phosphatidylserine (mechanism unknown).

$59 / 60 \bullet$ Sugiki/Prashek

Building on previous studies on intramolecular interactions within CERT [34], the phosphorylated form of the serine rich motif is shown to both bind the $\mathrm{PH}$ domain directly and to strongly enhance the interaction of the lipid transfer domain with the PH domain. Dephosphorylation of this motif allows each separate domain to function, and is supplemented by phosphorylation of the acidic tract of the FFAT motif, which strongly enhances VAP binding.

$66 \bullet \bullet$ Bean

Vps13p had previously been found to vaguely localise to many different sites in yeast, without any molecular understanding. By analysing the PX-domain-only protein Ypt35p, one of Vps13p's many reported interactors, a short linear motif "PxP" (in more detail: $\varnothing x \times \varnothing x P x P \emptyset x \emptyset$, where $\varnothing$ is hydrophobic) is identified not only in Ypt35p, but also in two other Vps13p interactors: Mcp1p on mitochondria, and Spo71p on forming spores (meiosis only). The region on $\mathrm{Vps13p}$ responsible overlaps with a region 
denoted DUF1162, and has multiple (6-8) all-beta repeats that contain a conserved $\varnothing \times \varnothing \times N$ sequence, which are widely conserved (e.g. humans), implying that interactors with PxP-like motifs may also be widespread.

$67 \bullet \bullet$ John Peter

The ability of Vps13p in presumed lipid transfer to/from mitochondria is separated from its function in endosomes. The bindings sites of both Vps13p and its mitochondrial receptor Mcp1p for each other are mapped approximately, and Mcp1 is shown to be more than just an anchor - it has two haem binding sites that might either be enzymes or form lipid binding cavities.

$68 \bullet \bullet$ Gonzalez Montoro

The vacuolar protein Vps39p is shown to bridge to mitochondria at vCLAMPs by binding Tom40p. Surprisingly, it is Vps39p's separate role in late endosome/vacuole formation that is required for genetic suppression of ER<->mitochondria lipid transfer defects by the Vps13p/Mcp1p pathway. Vps13p localises adjacent to, not within, extended VCLAMPs, suggesting that previous assumptions it bridges directly between vacuole and mitochondria are wrong.

1. Haj FG, Verveer PJ, Squire A, Neel BG, Bastiaens PI: Imaging sites of receptor dephosphorylation by PTP1B on the surface of the endoplasmic reticulum. Science 2002, 295:1708-1711.

2. Csordas G, Thomas AP, Hajnoczky G: Quasi-synaptic calcium signal transmission between endoplasmic reticulum and mitochondria. Embo J 1999, 18:96-108.

3. Taylor CW: Regulation of IP3 receptors by cyclic AMP. Cell Calcium 2017, 63:48-52.

4. Booth DM, Enyedi B, Geiszt M, Varnai P, Hajnoczky G: Redox Nanodomains Are Induced by and Control Calcium Signaling at the ER-Mitochondrial Interface. Mol Cell 2016, 63:240-248.

5. Sheftel AD, Zhang AS, Brown C, Shirihai OS, Ponka P: Direct interorganellar transfer of iron from endosome to mitochondrion. Blood 2007, 110:125-132.

6. Whatmore J, Wiedemann C, Somerharju P, Swigart P, Cockcroft S: Resynthesis of phosphatidylinositol in permeabilized neutrophils following phospholipase Cbeta activation: transport of the intermediate, phosphatidic acid, from the plasma membrane to the endoplasmic reticulum for phosphatidylinositol resynthesis is not dependent on soluble lipid carriers or vesicular transport. Biochem J 1999, 341 ( Pt 2):435-444.

7.•• Shai N, Yifrach E, van Roermund CWT, Cohen N, Bibi C, L IJ, Cavellini L, Meurisse J, Schuster R, Zada L, et al.: Systematic mapping of contact sites reveals tethers and a function for the peroxisome-mitochondria contact. Nat Commun 2018, 9:1761.

8. Kralt A, Carretta M, Mari M, Reggiori F, Steen A, Poolman B, Veenhoff LM: Intrinsically disordered linker and plasma membrane-binding motif sort Ist2 and Ssy1 to junctions. Traffic 2015, 16:135-147.

9. Perkins GA, Tjong J, Brown JM, Poquiz PH, Scott RT, Kolson DR, Ellisman MH, Spirou GA: The microarchitecture of mitochondria at active zones: electron tomography reveals novel anchoring scaffolds and cristae structured for high-rate metabolism. J Neurosci 2010, 30:1015-1026.

10. Olkkonen VM, Levine TP: Oxysterol binding proteins: in more than one place at one time? Biochem Cell Biol 2004, 82:87-98. 
11. Chung J, Torta F, Masai K, Lucast L, Czapla H, Tanner LB, Narayanaswamy P, Wenk MR, Nakatsu F, De Camilli P: Intracellular transport: PI4P/phosphatidylserine countertransport at ORP5- and ORP8-mediated ER-plasma membrane contacts. Science 2015, 349:428-432.

12. Huttlin EL, Ting L, Bruckner RJ, Gebreab F, Gygi MP, Szpyt J, Tam S, Zarraga G, Colby G, Baltier K, et al.: The BioPlex Network: A Systematic Exploration of the Human Interactome. Cell 2015, 162:425-440.

13. Hein MY, Hubner NC, Poser I, Cox J, Nagaraj N, Toyoda Y, Gak IA, Weisswange I, Mansfeld J, Buchholz $\mathrm{F}$, et al.: A human interactome in three quantitative dimensions organized by stoichiometries and abundances. Cell 2015, 163:712-723.

14. Furuita K, Jee J, Fukada H, Mishima M, Kojima C: Electrostatic interaction between oxysterolbinding protein and VAMP-associated protein A revealed by NMR and mutagenesis studies. J Biol Chem 2010, 285:12961-12970.

15. Kaiser SE, Brickner JH, Reilein AR, Fenn TD, Walter P, Brunger AT: Structural basis of FFAT motifmediated ER targeting. Structure 2005, 13:1035-1045.

16. Loewen CJ, Roy A, Levine TP: A conserved ER targeting motif in three families of lipid binding proteins and in Opi1p binds VAP. EMBO J 2003, 22:2025-2035.

17. Baron Y, Pedrioli PG, Tyagi K, Johnson C, Wood NT, Fountaine D, Wightman M, Alexandru G: VAPB/ALS8 interacts with FFAT-like proteins including the p97 cofactor FAF1 and the ASNA1 ATPase. BMC Biol 2014, 12:39.

18. Raiborg C, Wenzel EM, Pedersen NM, Olsvik H, Schink KO, Schultz SW, Vietri M, Nisi V, Bucci C, Brech A, et al.: Repeated ER-endosome contacts promote endosome translocation and neurite outgrowth. Nature 2015, 520:234-238.

19.• Di Mattia T, Wilhelm LP, Ikhlef S, Wendling C, Spehner D, Nomine Y, Giordano F, Mathelin C, Drin G, Tomasetto C, et al.: Identification of MOSPD2, a novel scaffold for endoplasmic reticulum membrane contact sites. EMBO Rep 2018, 19.

20. Mikitova V, Levine TP: Analysis of the key elements of FFAT-like motifs identifies new proteins that potentially bind VAP on the ER, including two AKAPs and FAPP2. PLoS One 2012, 7:e30455.

21. Dong R, Saheki Y, Swarup S, Lucast L, Harper JW, De Camilli P: Endosome-ER Contacts Control Actin Nucleation and Retromer Function through VAP-Dependent Regulation of PI4P. Cell 2016, 166:408-423.

22. Yagita Y, Shinohara K, Abe Y, Nakagawa K, Al-Owain M, Alkuraya FS, Fujiki Y: Deficiency of a Retinal Dystrophy Protein, Acyl-CoA Binding Domain-containing 5 (ACBD5), Impairs Peroxisomal betaOxidation of Very-long-chain Fatty Acids. J Biol Chem 2017, 292:691-705.

23. Costello JL, Castro IG, Schrader TA, Islinger M, Schrader M: Peroxisomal ACBD4 interacts with VAPB and promotes ER-peroxisome associations. Cell Cycle 2017, 16:1039-1045.

24. Hua R, Cheng D, Coyaud E, Freeman S, Di Pietro E, Wang Y, Vissa A, Yip CM, Fairn GD, Braverman N, et al.: VAPs and ACBD5 tether peroxisomes to the ER for peroxisome maintenance and lipid homeostasis. J Cell Biol 2017, 216:367-377.

25.•• Anding AL, Wang C, Chang TK, Sliter DA, Powers CM, Hofmann K, Youle RJ, Baehrecke EH: Vps13D Encodes a Ubiquitin-Binding Protein that Is Required for the Regulation of Mitochondrial Size and Clearance. Curr Biol 2018, 28:287-295 e286.

26.• Kumar N, Leonzino M, Hancock-Cerutti W, Horenkamp FA, Li P, Lees JA, Wheeler H, Reinisch KM, De Camilli P: VPS13A and VPS13C are lipid transport proteins differentially localized at ER contact sites. J Cell Biol 2018. 
27. De M, Oleskie AN, Ayyash M, Dutta S, Mancour L, Abazeed ME, Brace EJ, Skiniotis G, Fuller RS: The Vps13p-Cdc31p complex is directly required for TGN late endosome transport and TGN homotypic fusion. J Cell Biol 2017, 216:425-439.

28. Wong LH, Gatta AT, Levine TP: Lipid transfer proteins: the lipid commute by shuttles, bridges and tubes. Nature Reviews in Molecular Cell Biology 2018.

29. Strating JR, van Kuppeveld FJ: Viral rewiring of cellular lipid metabolism to create membranous replication compartments. Curr Opin Cell Biol 2017, 47:24-33.

30. Kumagai K, Elwell CA, Ando S, Engel JN, Hanada K: Both the $\mathrm{N}$ - and C- terminal regions of the Chlamydial inclusion protein $D(I n C D)$ are required for interaction with the pleckstrin homology domain of the ceramide transport protein CERT. Biochem Biophys Res Commun 2018, 505:1070-1076.

31. Stanhope R, Flora E, Bayne C, Derre I: IncV, a FFAT motif-containing Chlamydia protein, tethers the endoplasmic reticulum to the pathogen-containing vacuole. Proc Natl Acad Sci U S A 2017, 114:12039-12044.

32. Alpy F, Rousseau A, Schwab Y, Legueux F, Stoll I, Wendling C, Spiegelhalter C, Kessler P, Mathelin C, Rio MC, et al.: STARD3 or STARD3NL and VAP form a novel molecular tether between late endosomes and the ER. J Cell Sci 2013, 126:5500-5512.

33.• McCune BT, Tang W, Lu J, Eaglesham JB, Thorne L, Mayer AE, Condiff E, Nice TJ, Goodfellow I, Krezel AM, et al.: Noroviruses Co-opt the Function of Host Proteins VAPA and VAPB for Replication via a Phenylalanine-Phenylalanine-Acidic-Tract-Motif Mimic in Nonstructural Viral Protein NS1/2. MBio 2017, 8.

34. Kumagai K, Kawano-Kawada M, Hanada K: Phosphoregulation of the ceramide transport protein CERT at serine 315 in the interaction with VAMP-associated protein (VAP) for inter-organelle trafficking of ceramide in mammalian cells. J Biol Chem 2014, 289:10748-10760.

35. Lim ST, Antonucci DE, Scannevin RH, Trimmer JS: A novel targeting signal for proximal clustering of the Kv2.1 K+ channel in hippocampal neurons. Neuron 2000, 25:385-397.

36. Zhang Y, McKay SE, Bewley B, Kaczmarek LK: Repetitive firing triggers clustering of Kv2.1 potassium channels in Aplysia neurons. J Biol Chem 2008, 283:10632-10641.

37. Misonou H, Mohapatra DP, Trimmer JS: Kv2.1: a voltage-gated k+ channel critical to dynamic control of neuronal excitability. Neurotoxicology 2005, 26:743-752.

38. Fox PD, Haberkorn CJ, Akin EJ, Seel PJ, Krapf D, Tamkun MM: Induction of stable ER-plasmamembrane junctions by Kv2.1 potassium channels. J Cell Sci 2015, 128:2096-2105.

39. Park KS, Mohapatra DP, Misonou H, Trimmer JS: Graded regulation of the Kv2.1 potassium channel by variable phosphorylation. Science 2006, 313:976-979.

40.•• Johnson B, Leek AN, Sole L, Maverick EE, Levine TP, Tamkun MM: Kv2 potassium channels form endoplasmic reticulum/plasma membrane junctions via interaction with VAPA and VAPB. Proc Natl Acad Sci U S A 2018, 115:E7331-E7340.

41.•• Kirmiz M, Vierra NC, Palacio S, Trimmer JS: Identification of VAPA and VAPB as Kv2 ChannelInteracting Proteins Defining Endoplasmic Reticulum-Plasma Membrane Junctions in Mammalian Brain Neurons. J Neurosci 2018, 38:7562-7584.

42. Schluter K, Waschbusch D, Anft M, Hugging D, Kind S, Hanisch J, Lakisic G, Gautreau A, Barnekow A, Stradal TE: JMY is involved in anterograde vesicle trafficking from the trans-Golgi network. Eur J Cell Biol 2014, 93:194-204. 
43. Garcia-Santamarina S, Boronat S, Hidalgo E: Reversible cysteine oxidation in hydrogen peroxide sensing and signal transduction. Biochemistry 2014, 53:2560-2580.

44. Murphy SE, Levine TP: VAP, a Versatile Access Point for the Endoplasmic Reticulum: Review and analysis of FFAT-like motifs in the VAPome. Biochim Biophys Acta 2016, 1861:952-961.

45. Felberbaum R, Wilson NR, Cheng D, Peng J, Hochstrasser M: Desumoylation of the endoplasmic reticulum membrane VAP family protein Scs2 by Ulp1 and SUMO regulation of the inositol synthesis pathway. Mol Cell Biol 2012, 32:64-75.

46. Murray LA, Sheng X, Cristea IM: Orchestration of protein acetylation as a toggle for cellular defense and virus replication. Nat Commun 2018, 9:4967.

47. Heo WD, Inoue T, Park WS, Kim ML, Park BO, Wandless TJ, Meyer T: PI(3,4,5)P3 and PI(4,5)P2 lipids target proteins with polybasic clusters to the plasma membrane. Science 2006, 314:1458-1461.

48.•• Lees JA, Messa M, Sun EW, Wheeler H, Torta F, Wenk MR, De Camilli P, Reinisch KM: Lipid transport by TMEM24 at ER-plasma membrane contacts regulates pulsatile insulin secretion. Science 2017, 355.

49. Bigay J, Antonny B: Curvature, lipid packing, and electrostatics of membrane organelles: defining cellular territories in determining specificity. Dev Cell 2012, 23:886-895.

50. Drin G, Antonny B: Amphipathic helices and membrane curvature. FEBS Lett 2010, 584:1840-1847.

51. Olmos Y, Perdrix-Rosell A, Carlton JG: Membrane Binding by CHMP7 Coordinates ESCRT-IIIDependent Nuclear Envelope Reformation. Curr Biol 2016, 26:2635-2641.

52. Markgraf DF, Klemm RW, Junker M, Hannibal-Bach HK, Ejsing CS, Rapoport TA: An ER protein functionally couples neutral lipid metabolism on lipid droplets to membrane lipid synthesis in the ER. Cell Rep 2014, 6:44-55.

53. Maass K, Fischer MA, Seiler M, Temmerman K, Nickel W, Seedorf M: A signal comprising a basic cluster and an amphipathic alpha-helix interacts with lipids and is required for the transport of Ist2 to the yeast cortical ER. J Cell Sci 2009, 122:625-635.

54. Swaney DL, Beltrao P, Starita L, Guo A, Rush J, Fields S, Krogan NJ, Villen J: Global analysis of phosphorylation and ubiquitylation cross-talk in protein degradation. Nat Methods 2013, 10:676-682.

55. Korzeniowski MK, Manjarres IM, Varnai P, Balla T: Activation of STIM1-Orai1 involves an intramolecular switching mechanism. Sci Signal 2010, 3:ra82.

56. Hirve N, Rajanikanth V, Hogan PG, Gudlur A: Coiled-Coil Formation Conveys a STIM1 Signal from ER Lumen to Cytoplasm. Cell Rep 2018, 22:72-83.

57.•• Bian X, Saheki Y, De Camilli P: Ca(2+) releases E-Syt1 autoinhibition to couple ER-plasma membrane tethering with lipid transport. EMBO J 2018, 37:219-234.

58. Kumagai K, Kawano M, Shinkai-Ouchi F, Nishijima M, Hanada K: Interorganelle trafficking of ceramide is regulated by phosphorylation-dependent cooperativity between the $\mathrm{PH}$ and START domains of CERT. J Biol Chem 2007, 282:17758-17766.

59.• Sugiki T, Egawa D, Kumagai K, Kojima C, Fujiwara T, Takeuchi K, Shimada I, Hanada K, Takahashi H: Phosphoinositide binding by the PH domain in ceramide transfer protein (CERT) is inhibited by hyperphosphorylation of an adjacent serine-repeat motif. J Biol Chem 2018, 293:11206-11217.

60.•• Prashek J, Bouyain S, Fu M, Li Y, Berkes D, Yao X: Interaction between the PH and START domains of ceramide transfer protein competes with phosphatidylinositol 4-phosphate binding by the PH domain. J Biol Chem 2017, 292:14217-14228. 
61. Saito S, Matsui H, Kawano M, Kumagai K, Tomishige N, Hanada K, Echigo S, Tamura S, Kobayashi T: Protein phosphatase 2Cepsilon is an endoplasmic reticulum integral membrane protein that dephosphorylates the ceramide transport protein CERT to enhance its association with organelle membranes. J Biol Chem 2008, 283:6584-6593.

62. Mesmin B, Bigay J, Moser von Filseck J, Lacas-Gervais S, Drin G, Antonny B: A four-step cycle driven by $\mathrm{PI}(4) \mathrm{P}$ hydrolysis directs sterol/PI(4)P exchange by the ER-Golgi tether OSBP. Cell 2013, 155:830-843.

63. Weber-Boyvat M, Kentala H, Lilja J, Vihervaara T, Hanninen R, Zhou Y, Peranen J, Nyman TA, Ivaska J, Olkkonen VM: OSBP-related protein 3 (ORP3) coupling with VAMP-associated protein A regulates R-Ras activity. Exp Cell Res 2015, 331:278-291.

64. Saita S, Shirane M, Natume T, lemura S, Nakayama KI: Promotion of neurite extension by protrudin requires its interaction with vesicle-associated membrane protein-associated protein. $J$ Biol Chem 2009, 284:13766-13777.

65. Park JS, Thorsness MK, Policastro R, McGoldrick LL, Hollingsworth NM, Thorsness PE, Neiman AM: Yeast Vps13 promotes mitochondrial function and is localized at membrane contact sites. Mol Biol Cell 2016, 27:2435-2449.

66.• Bean BDM, Dziurdzik SK, Kolehmainen KL, Fowler CMS, Kwong WK, Grad LI, Davey M, Schluter C, Conibear E: Competitive organelle-specific adaptors recruit Vps13 to membrane contact sites. J Cell Biol 2018, 217:3593-3607.

67.•• John Peter AT, Herrmann B, Antunes D, Rapaport D, Dimmer KS, Kornmann B: Vps13-Mcp1 interact at vacuole-mitochondria interfaces and bypass ER-mitochondria contact sites. $J$ Cell Biol 2017, 216:3219-3229.

68.•• Gonzalez Montoro A, Auffarth K, Honscher C, Bohnert M, Becker T, Warscheid B, Reggiori F, van der Laan M, Frohlich F, Ungermann C: Vps39 Interacts with Tom40 to Establish One of Two Functionally Distinct Vacuole-Mitochondria Contact Sites. Dev Cell 2018, 45:621-636 e627.

69. Ladinsky MS, Mastronarde DN, McIntosh JR, Howell KE, Staehelin LA: Golgi structure in three dimensions: functional insights from the normal rat kidney cell. J Cell Biol 1999, 144:11351149.

70. Chowdhury S, Otomo C, Leitner A, Ohashi K, Aebersold R, Lander GC, Otomo T: Insights into autophagosome biogenesis from structural and biochemical analyses of the ATG2A-WIPI4 complex. Proc Natl Acad Sci U S A 2018, 115:E9792-E9801. 


\section{Figure 1}

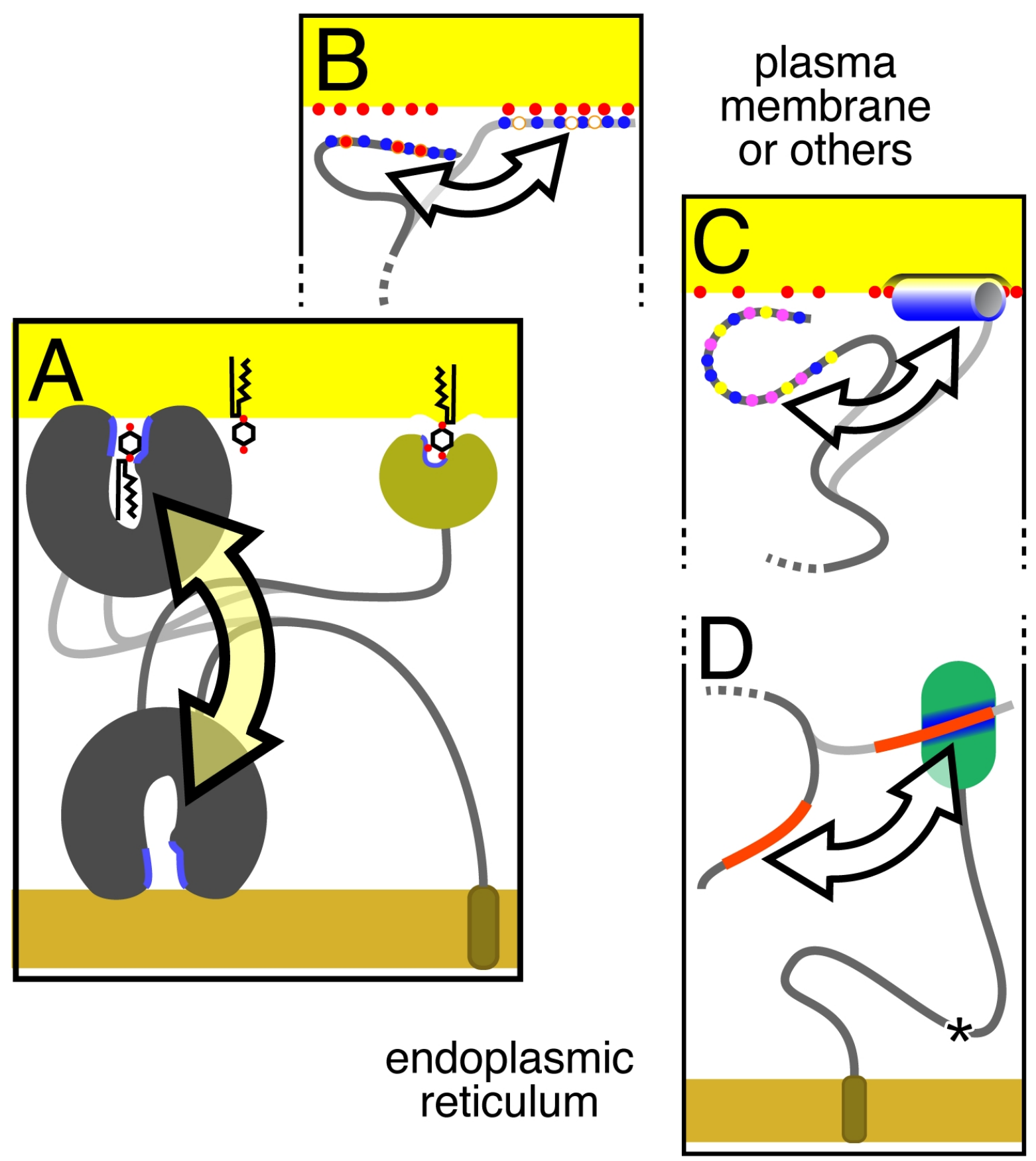




\section{Figure 2}

A OSBP

RdgB

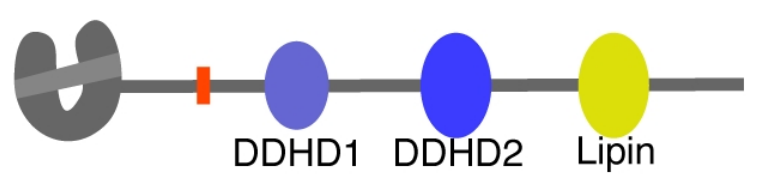

B

CERT

FAF1

Protrudin

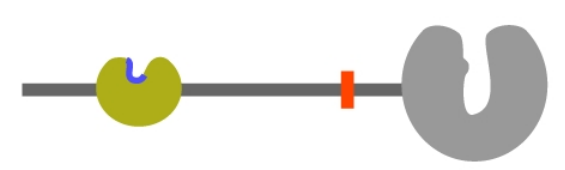

DDHD1 DDHD2 Lipin

CERT

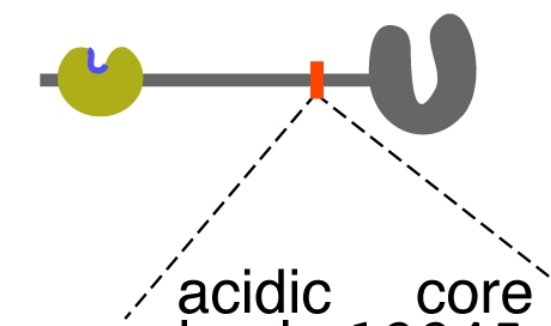
tract $12345 \times 7$ SLINEEFFEAP司 SDSDGDDFEDT EAEPDE품소봅
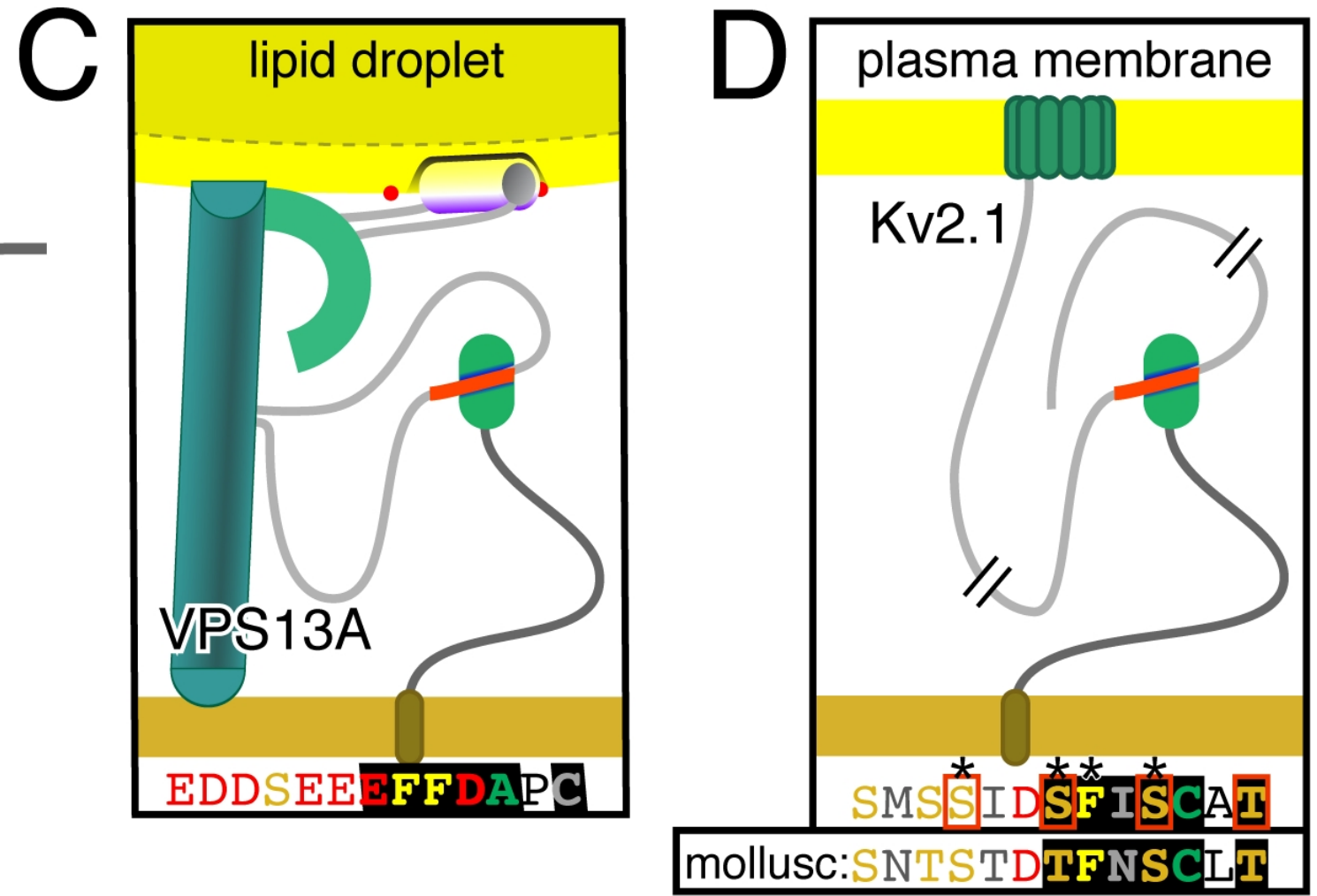


\section{Figure 3}

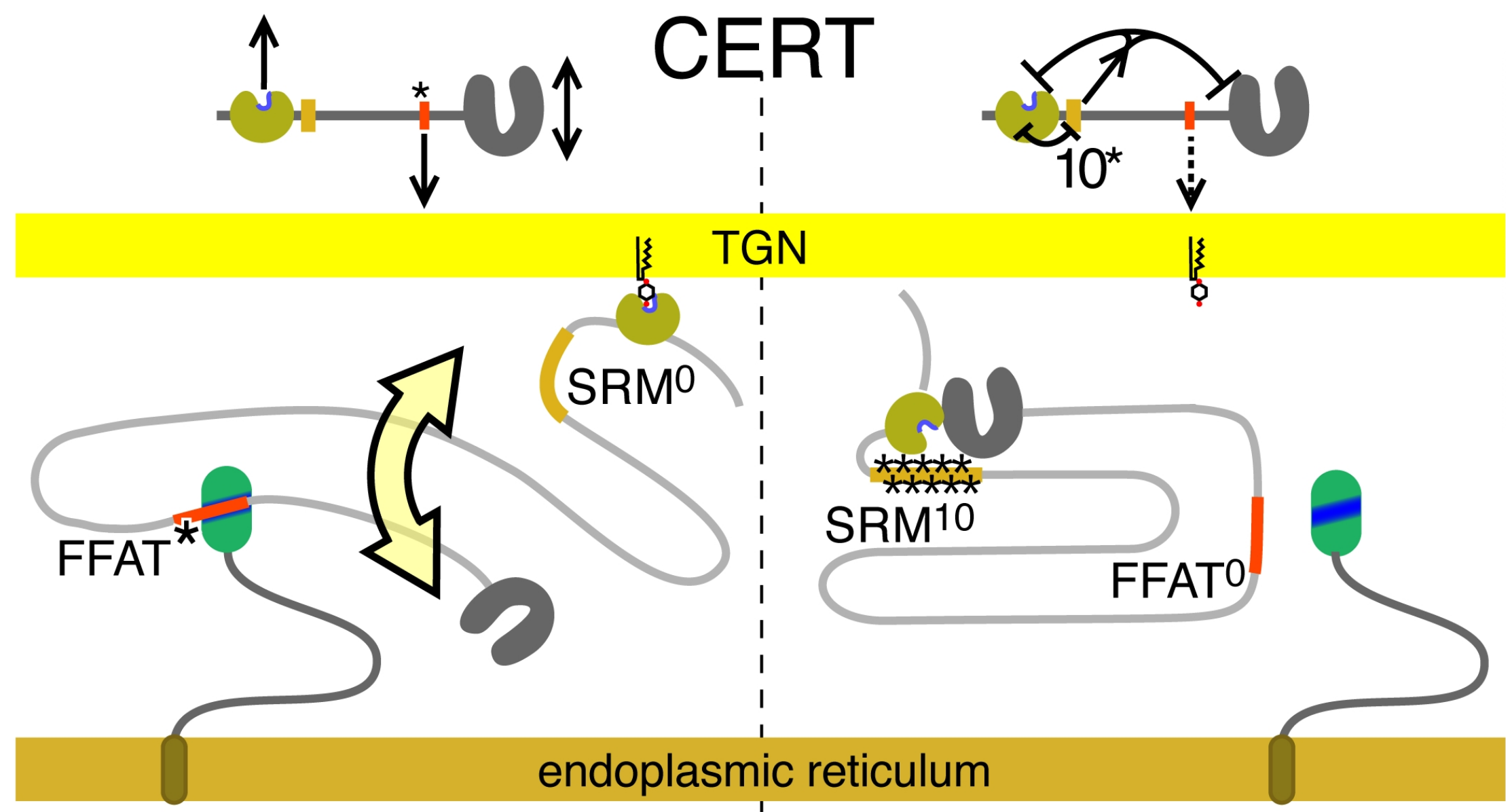

active / open 


\section{Figure 4}

A

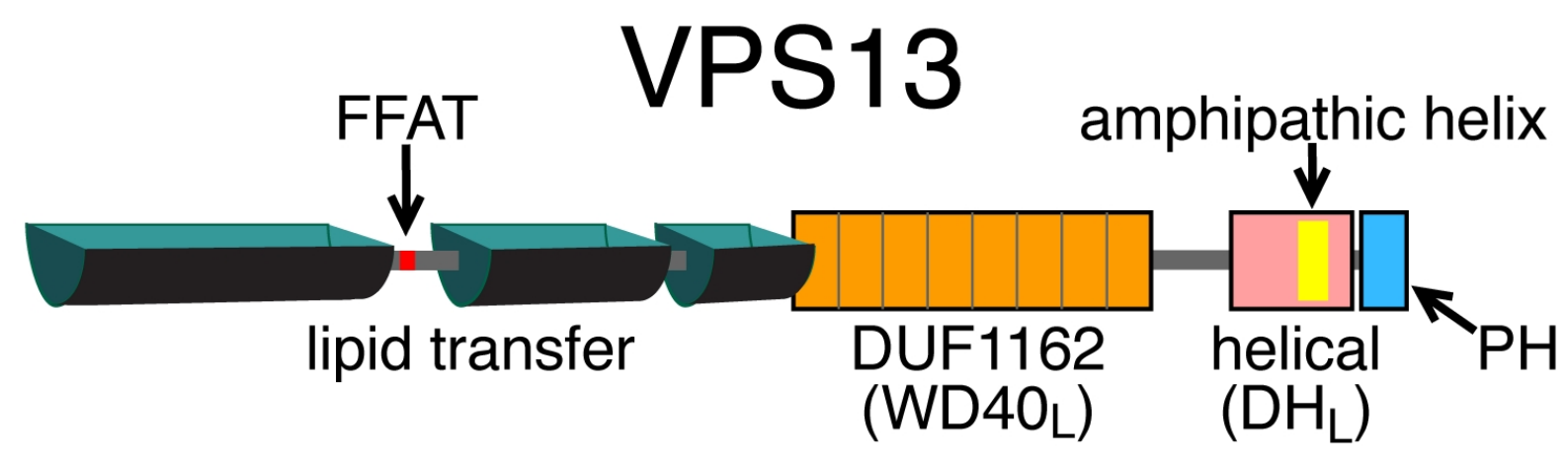

B

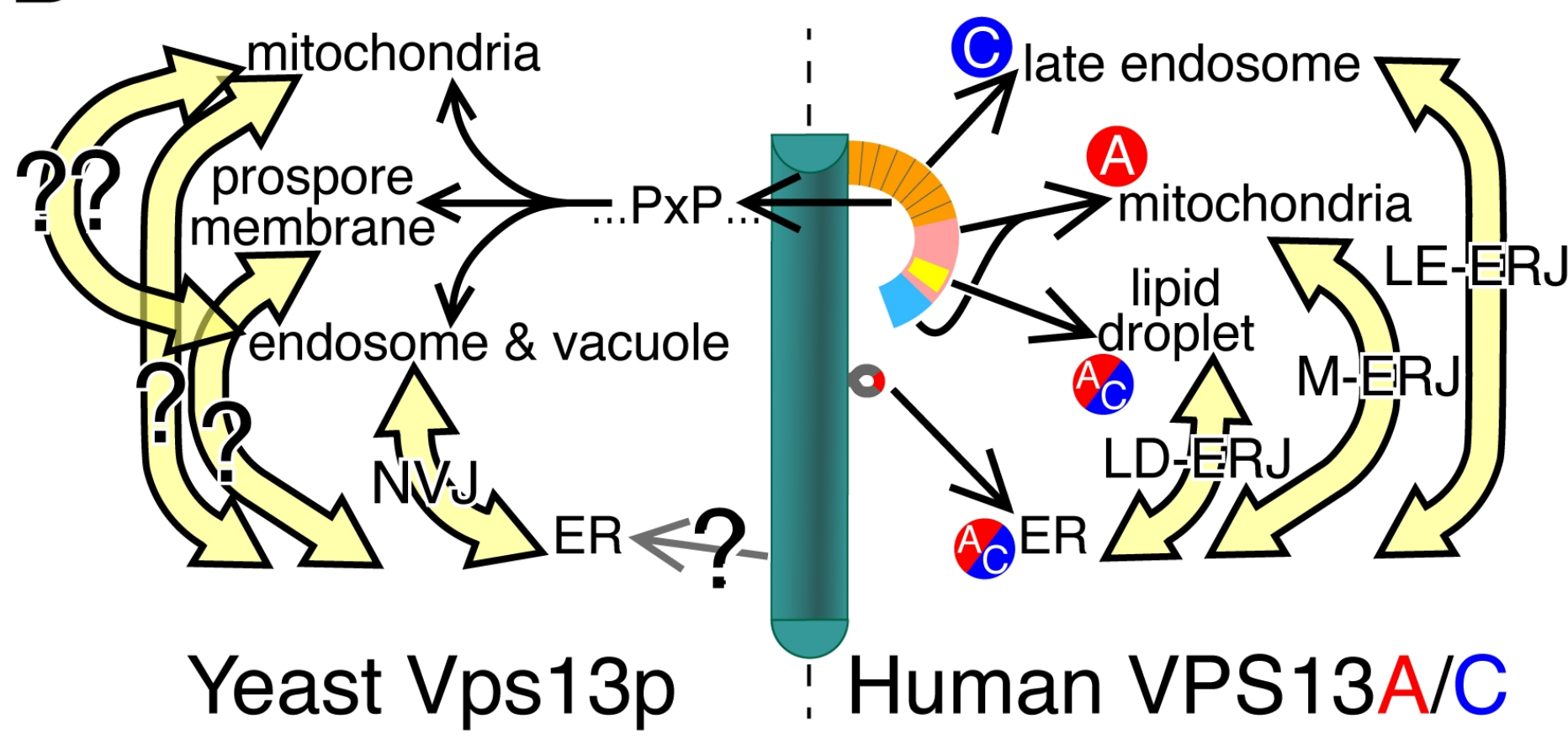

